\title{
La surexploitation des eaux souterraines : enjeux et gouvernance
}

\author{
Olivier Petit \\ Économiste, EREIA / C3ED, UMR nº 063 IRD-UVSQ, Université d'Artois, 9 rue du Temple, BP 665, 62030 Arras cedex, France
}

Le devenir des eaux souterraines constitue l'un des axes majeurs de la réflexion sur la gestion durable de la ressource en eau. Olivier Petit porte ici un regard d'économiste sur la question. Après avoir mis en perspective toute l'acuité du problème de la surexploitation locale de certains aquifères par l'irrigation et tout le flou qui entoure la définition de ce terme, l'auteur montre que la réponse des politiques de l'eau est longtemps restée insatisfaisante par incapacité à transcender deux visions totalement antagonistes. La première suggère que l'instauration de droits de propriété privés et l'établissement d'un marché de l'eau est l'unique manière d'éviter les conflits d'usage. La seconde affirme que seul l’État est capable de garantir une gestion raisonnée et une répartition équitable de l'eau. En s'appuyant sur des fondements théoriques variés, Olivier Petit propose ici une troisième voie, la "gouvernance de l'eau», basée sur la combinaison entre une coordination des actions individuelles régulée par un marché, des actions collectives d'intérêt public impulsées par l'État et, enfin, des actions collectives à caractère communautaire protégeant les intérêts de l'ensemble des usagers.

La Rédaction

\section{Mots-clés :}

eaux souterraines ; surexploitation; gouvernance ; économie des ressources en eau

\section{Keywords: groundwater resources; overexploitation; governance; water resources economics}

Résumé - Cet article aborde les enjeux posés par la surexploitation des eaux souterraines à travers le monde dans un contexte de raréfaction des ressources en eau douce. Loin de constituer un phénomène généralisé, la surexploitation touche les eaux souterraines dans les régions aux climats semi-arides et arides, mais également dans les régions aux climats tempérés. De fait, depuis la fin de la Seconde Guerre mondiale, le développement de l'irrigation et les révolutions vertes ont grandement contribué aux crises d'approvisionnement. La lutte contre la surexploitation des eaux souterraines est assimilable à une gouvernance durable de ces ressources. En tentant de dépasser la dichotomie entre intervention publique (réglementation et taxes) et marchandisation (mise en place de marchés d'eau souterraine), notre analyse de la gouvernance des eaux souterraines montre que celle-ci repose aujourd'hui davantage sur un compromis entre la coordination des actions individuelles (approche marchande) et les formes de l'action collective (action collective d'intérêt public et action collective d'intérêt communautaire).

\begin{abstract}
Groundwater overexploitation: Stakes and governance. Groundwater resources have long been considered as inexhaustible ones. Far from constituting a generalized phenomenon, overexploitation of groundwater resources is observable in the semi-arid and arid climates areas, but also in the areas with temperate climates. In fact, since the end of the Second World War, the development of irrigation and the green revolutions have largely contributed to the crises of provisioning. In order to prevent overexploitation and conflicts between water users, two main solutions have been traditionally proposed: market or state regulation. Our analysis tries to go beyond this dichotomy between public intervention (regulation and taxes) and market-oriented approaches (implementation of groundwater markets). A more pragmatic look at reality shows that water markets are not as efficient as many authors claim, because market failures and transaction costs are inherent to the market allocation of groundwater resources. Moreover, state management of groundwater resources doesn't seem to be efficient, because of a lack of enforcement and of a clear definition of property rights. But empirical studies have shown that a shift has occurred in the allocation and management of groundwater resources. This shift shows the importance to take into account water resource users in the dynamics of collective action. Our analysis of the governance of groundwater resources identifies the existence of a compromise between the coordination of the individual actions (market-oriented approach) and the forms of collective action (collective action for public interest and collective action for community interest).
\end{abstract}

Auteur correspondant : opetitfr@yahoo.fr 
Les eaux souterraines font partie d'une catégorie de ressources difficiles à appréhender. Elles relèvent typiquement des biens d'environnement et semblent appartenir à tout le monde, en même temps qu'elles n'appartiennent à personne.

On s'est pendant longtemps contenté de cette ambiguïté juridique, en laissant les usagers s'approprier cette ressource et l'exploiter au gré de leurs besoins. Mais, depuis la seconde moitié du XXe siècle, la pression démographique, le développement de l'irrigation et de l'agriculture intensive ont mis cette ressource en danger. Les propositions visant à limiter les impacts des prélèvements excessifs ont alors pris essentiellement deux voies.

Ces deux options s'appuient, pour la plupart, sur une lecture de The Tragedy of the Commons, le texte d'Hardin (1968). Cet auteur affirme que les ressources communes seraient vouées inexorablement à l'épuisement car chacun exploite la ressource, en faisant supporter aux autres usagers les coûts de cette exploitation. Il s'ensuivrait une course à l'exploitation qui ne pourrait être résolue que par la mise en place de droits de propriété - privés ou publics. Cette alternative a donné lieu à deux types de recommandations de la part des économistes.

Pour certains, la définition de droits de propriété privés et l'établissement d'un marché sur les ressources naturelles permettraient d'éviter les conflits d'usage, de parvenir à une exploitation efficace des ressources et d'éviter leur surexploitation par la détermination d'un prix s'imposant à tous. En contraste, d'autres affirment que l'État pourrait éviter la surexploitation, résoudre les conflits d'usage et opérer une répartition équitable des ressources, en tant que garant de l'intérêt général.

Mais cette alternative ne semble pas correspondre aux situations observables. Il existe en fait une très grande variété de situations reposant conjointement sur l'échange marchand, l'intervention publique ou sur des procédures mises en place par les acteurs eux-mêmes, qui permettent de surmonter la tragédie décrite par Hardin. Or, les économistes ont longtemps délaissé l'étude de ces voies car la plupart d'entre eux confondent la propriété commune avec le libre accès ${ }^{1}$.

Nous souhaitons, dans le cadre de cet article, mettre en lumière les éléments de débat qui permettent d'interroger le problème de la surexploitation des eaux souterraines dans une perspective allant au-delà de la dichotomie habituellement retenue. Pour ce faire, nous soulignons d'abord les raisons pour lesquelles la surexploitation des eaux souterraines est devenue un sujet de préoccupation croissant. Puis, nous engageons une discussion sur le concept de surexploitation appliqué aux eaux souterraines. Ceci nous permet enfin de porter un regard critique sur les arguments avancés afin de relever

\footnotetext{
1 Pour une clarification du concept de propriété commune, voir en particulier Ciriacy-Wantrup et Bishop (1975), AguileraKlink (1994), ou encore Weber et Réveret (1994).
}

les risques d'une interprétation abusive des intérêts et des limites de l'approche marchande et de l'intervention publique, puis de fonder une approche plus pragmatique de la gouvernance des eaux souterraines ${ }^{2}$.

\section{L'eau souterraine : une richesse à préserver}

Longtemps considérées comme inépuisables, les ressources en eau font l'objet d'une attention accrue de la part de la communauté internationale qui souligne, à juste titre, le caractère stratégique et emblématique d'une gestion durable de ces ressources.

L'eau est pourtant abondante, mais sa répartition sur la terre est largement inégalitaire. Certains pays souffrent d'un manque d'eau important pour assurer les besoins vitaux élémentaires de leurs populations, tandis que d'autres ne savent plus comment maîtriser le trop plein d'eau chronique qui envahit progressivement les terres arables et déplace des flux entiers de population. Les réserves en eau douce de la planète ne constituent qu'une infime partie de l'eau présente sur terre (tout juste $3 \%$ ). Or, si l'on considère que près de $70 \%$ de cette eau douce reste emprisonnée dans la glace et dans les neiges et que l'eau contenue dans l'atmosphère, les rivières et les lacs représente moins d'1 \% du stock d'eau douce, on comprend pourquoi les eaux souterraines (99\% du stock d'eau douce continentale) sont une richesse essentielle à préserver (Shiklomanov, 1993). Cependant, ce relevé des stocks ne reflète pas les flux globaux de ressources renouvelables. Notons que les eaux souterraines représentent environ un tiers de l'écoulement global des eaux continentales (de l'ordre de $13000 \mathrm{~km}^{3}$ /an pour un total d'environ $43000 \mathrm{~km}^{3} / \mathrm{an}$ ), ce qui permet à ces ressources de soutenir le débit des cours d'eau superficiels (Encadré 1). La baisse du niveau piézométrique des eaux souterraines peut donc provoquer l'assèchement temporaire de certains cours d'eau exutoires (cas de la Conie, exutoire à la nappe de Beauce qui s'est trouvé asséché au milieu des années 1990).

\footnotetext{
${ }^{2}$ Cet article est issu d'une thèse de doctorat en sciences économiques intitulée «De la coordination des actions individuelles aux formes de l'action collective : une exploration des modes de gouvernance des eaux souterraines ", réalisée sous la direction du professeur S. Faucheux et soutenue en décembre 2002 à l'Université de Versailles Saint-Quentin-en-Yvelines. Par ailleurs, certains des éléments discutés dans ce texte ont été élaborés dans le cadre d'un contrat de recherches, «Appropriation sociale du problème de gestion durable de la nappe de Beauce ", du Programme inter-institutionnel de recherches et d'études en économie de l'environnement (PIREE) du ministère de l'Aménagement du territoire et de l'Environnement contrat $n^{\circ} 99123$ - sous la direction scientifique du professeur M. O'Connor et en collaboration avec B. Corcos, rapport final août 2001.
} 


\begin{abstract}
Encadré 1. Quelques éléments sur le fonctionnement d'un aquifère rechargeable
Il est utile tout d'abord de distinguer les eaux souterraines (le contenu) des aquifères (qui forment le contenant). Rappelons qu'un aquifère est une « formation géologique constituée de roches perméables (formations poreuses et/ou fissurées) comportant une zone saturée - ensemble du milieu solide et de l'eau contenue - suffisamment conductrice d'eau souterraine pour permettre l'écoulement significatif d'une nappe souterraine et le captage (drainage, pompage...) de quantités d'eau appréciables. Un aquifère libre comporte une surface libre et une zone non saturée (en eau). Un aquifère captif est entièrement saturé, comportant une nappe captive (sans surface libre ni zone non saturée), délimité au toit par des formations à perméabilité très faible faisant obstacle à tout flux appréciable » (Adour Garonne, 2002, p. 81).

Pour comprendre le fonctionnement d'un aquifère rechargeable, il est important de distinguer les entrées (recharges) des sorties (fuites et prélèvements) de l'aquifère. Les eaux souterraines sont dans la plupart des cas rechargées par les pluies hivernales. On parle à ce sujet de "pluies efficaces » pour désigner la quantité d'eau de pluie s'infiltrant dans l'aquifère. Les autres composantes de la recharge des nappes sont constituées par les quantités d'eau irriguées ou drainées qui ne sont pas consommées par les plantes et qui ne repartent pas dans l'atmosphère. La végétation, la pente des sols et les roches constituant les formations géologiques de l'aquifère conditionnent le rythme de remplissage et la vitesse de circulation de l'eau entre les différentes couches géologiques. Du point de vue des sorties de la nappe, outre les prélèvements effectués pour les différents usages (alimentation en eau potable et autres usages domestiques, irrigation et usages industriels essentiellement), les fuites de l'aquifère vers les rivières exutoires à la nappe peuvent représenter des flux au moins aussi importants que les prélèvements effectués par l'homme. Une difficulté apparaît pour offrir une mesure de ces quantités d'eau qui s'écoulent des différents exutoires, car le flux dépend d'un grand nombre de facteurs et notamment du niveau de remplissage de la nappe.
\end{abstract}

De surcroît, cette situation de pénuries chroniques risque de s'aggraver à mesure que la population mondiale s'élève et que le rythme de renouvellement des stocks d'eau douce est mis en danger. Les perspectives de croissance de la consommation en eau sont en effet inquiétantes et, si la tendance estimée sur le siècle dernier (multiplication par 10 de la consommation mondiale d'eau) se poursuit, la pénurie pourrait toucher l'ensemble des continents ${ }^{3}$. Les incertitudes climatiques sont encore accrues si l'on considère un horizon de temps plus long. Dans ce cas, les impacts des changements climatiques sur la disponibilité de la ressource en eau et la pression exercée par l'accroissement démographique global pourraient encore accroître les disparités régionales.

L'eau souterraine fait partie de cette catégorie de ressources difficiles à appréhender car invisible tant qu'elle n'est pas extraite. Si des efforts considérables ont été fournis ces dernières années pour estimer les quantités d'eau présentes dans les aquifères, la préoccupation touchant à l'allocation et à la gestion de ces ressources entre les différents usages n'a émergé que récemment ${ }^{4}$, suite à une prise de conscience de la nécessité de protéger la qualité et d'assurer la pérennité de l'approvisionnement en eau au plus grand nombre. Cette préoccupation n'est pas sans lien avec les conflits d'usage qui se sont manifestés, ni avec la possibilité technique de mesurer les incidences de l'activité humaine sur l'état des ressources en eau.

\footnotetext{
${ }^{3}$ D’après les estimations effectuées par Shiklomanov (1991), la consommation annuelle d'eau dans le monde s'élevait à $579 \mathrm{~km}^{3}$ en 1900 et devait atteindre $5190 \mathrm{~km}^{3}$ en l'an 2000.

${ }^{4}$ Une exception notable, qui constitue un travail précurseur, est le travail d'Ollagnon (voir notamment Ollagnon, 1989), qui a conduit dès le milieu des années 1970 une étude des modes d'appropriation des eaux souterraines sur la nappe d'Alsace, dans une optique de gestion patrimoniale. Nous reviendrons plus loin sur ce travail.
}

Désormais, les eaux souterraines sont reconnues dans l'ensemble de leurs dimensions : support de vie des écosystèmes (elles permettent notamment la conservation des zones humides et de leur riche biodiversité), elles assurent également le maintien du flux des cours d'eau exutoires. Enfin, généralement de meilleure qualité que les eaux de surface, les eaux souterraines contribuent à une large part de l'alimentation en eau potable et permettent l'essor d'une activité économique (en particulier dans les secteurs agricoles et industriels) dont le développement est conditionné par la qualité comme par la quantité de ressources disponibles.

Parmi ces activités économiques, l'irrigation demeure largement tributaire des eaux souterraines, surtout dans les régions aux climats arides et semi-arides où la disponibilité en eaux de surface est restreinte. Ainsi, l'exploitation des eaux souterraines est un facteur crucial de développement économique et social d'une région. Foster (2000) affirme que des forages ont été pratiqués pour extraire de l'eau du sous-sol depuis la préhistoire. Mais l'exploitation des aquifères à grande échelle est un phénomène qui s'est surtout développé durant la deuxième moitié du XXe siècle. Cette exploitation a parfois été encouragée par les gouvernements qui voyaient dans le développement de l'irrigation un moyen de satisfaire les besoins des populations rurales, ainsi qu'une voie pour accroître leur popularité, sans se soucier des caractéristiques propres au fonctionnement des aquifères. Le progrès technique, couplé avec la croissance du nombre de forages pratiqués, souvent de manière anarchique, a considérablement accru la fragilité de l'équilibre entre recharge naturelle et prélèvements d'origine anthropique. Tandis que les eaux souterraines pouvaient paraître une ressource disponible en quantité infinie lorsque les techniques de prélèvements étaient encore archaïques, 
la mécanisation et l'effort consenti par les gouvernements pour subventionner l'accès à l'énergie et permettre l'utilisation de pompes de plus en plus puissantes allaient progressivement conduire les utilisateurs à se soucier des problèmes de surexploitation et à mesurer la finitude des réserves en eaux souterraines. Grellet (2001, p. 318) affirme l'importance de cette mutation technique, notamment dans les pays en développement :

«À côté de ces systèmes d'irrigation traditionnels, que nous appellerons «de surface», est apparue dans la seconde moitié $\mathrm{du} X \mathrm{X}^{\mathrm{e}}$ siècle une irrigation de pompage, rendue possible par des motopompes électriques ou à fuel. Ces motopompes ont joué un rôle essentiel dans les Révolutions vertes, fortes consommatrices d'eau, puisqu'elles ont permis d'obtenir rapidement de l'eau dans des zones sèches qui ne disposaient pas des infrastructures nécessaires à l'irrigation de surface. »

Cette mutation s'est accomplie à différentes époques suivant le rythme de développement des pays, mais on peut considérer que les décennies 1960 et 1970 ont marqué un profond changement dans l'exploitation des eaux souterraines à grande échelle. La responsabilité des gouvernements dans cette situation peut se mesurer à l'aune des objectifs dévolus aux organismes chargés de promouvoir le développement agricole et l'exploitation des eaux souterraines. Pour prendre deux exemples assez significatifs, les États-Unis et l'Inde ont créé des structures publiques en charge du développement agricole et de l'administration des ressources en eau. Le mandat initial de ces organismes était de favoriser et de promouvoir l'exploitation des ressources en eau ${ }^{5}$, non de les protéger ou de les gérer.

Les situations de surexploitation des eaux souterraines demeurent aujourd'hui encore un phénomène localisé qui ne touche pas l'ensemble des ressources d'un pays. On ne peut donc parler à ce sujet d'un phénomène généralisé, car le rythme d'exploitation est différent d'un pays à un autre, d'une région à une autre.

Selon des données du ministère indien des Ressources en eau, seules $32 \%$ des ressources en eaux souterraines potentiellement utilisables annuellement sont exploitées. La situation est néanmoins contrastée suivant les États et les aquifères. Ainsi, ce ratio serait de $94 \%$ au Punjab et de $84 \%$ en Haryana, faisant craindre une surexploitation prochaine à mesure que les quantités d'eau extraites s'accroissent. À un niveau plus local en revanche, six districts au Punjab et quatre en Haryana auraient un taux d'exploitation supérieur à 140 \%. Saleth (1996) rapporte le cas du district de Kapurthala où le ratio serait même de $259 \%$.

Cette situation nous permet de souligner que la surexploitation des aquifères est un phénomène qui ne

\footnotetext{
${ }^{5}$ On parle d'ailleurs plutôt de développement, mais cette notion renvoie bien à celle d'exploitation.
}

touche pas que les pays aux climats arides et semi-arides. L'Agence européenne de l'environnement rapporte ainsi que 11 pays européens au moins connaissent des problèmes de surexploitation de leurs eaux souterraines et que ceci se traduit dans 9 cas sur 11 par des intrusions salines (European Environment Agency, 1999b). Une enquête menée par ce même organisme révèle que, sur un échantillon de 200 zones humides recensées en Europe, 11 d'entre elles sont menacées par l'extinction d'espèces, extinction qui serait liée à la surexploitation des aquifères (European Environment Agency, 1999a).

Face à une telle mutation, il est devenu nécessaire d'envisager l'exploitation des eaux souterraines dans la perspective d'un développement durable. Cette préoccupation pose cependant des problèmes touchant à la dimension polysémique de ce concept et au seuil à partir duquel une ressource peut être considérée comme surexploitée.

\section{La surexploitation des aquifères : un concept ambigu}

La notion de surexploitation des aquifères ${ }^{6}$ est au centre d'un débat scientifique qui a connu un essor dans le courant des années 1970. Cette notion est appliquée essentiellement dans des situations climatiques arides et semi-arides; et plus particulièrement dans les régions où l'irrigation à grande échelle des cultures s'est développée. Suivant le point de vue adopté et la hiérarchisation des priorités affichées, la notion de surexploitation ne renverra pas aux mêmes définitions. Il n'existe d'ailleurs à strictement parler aucune définition rigoureuse de cette notion. Ceci tient à un certain nombre de facteurs affectant le point de vue adopté. En effet, sur quelle superficie le bilan entre entrées et sorties de l'aquifère doit-il être évalué? Sur quelle période est-il nécessaire de prendre en considération ce bilan : sur une année hydrique, une décennie, un siècle? Comment tenir compte des incertitudes inhérentes au cycle de l'eau et de la difficulté même qui subsiste pour évaluer les quantités entrant dans l'aquifère sans même parler du stock global de ressources disponibles? Malgré ces limites, la notion de surexploitation des aquifères renvoie généralement à une situation où les quantités extraites sont estimées être plus importantes que la recharge de la nappe. Ainsi que le soulignent Hernandez-Mora et al. (2001),

\footnotetext{
${ }^{6}$ Dans la littérature anglo-saxonne, on parle aussi souvent de groundwater overexploitation, overdraft, overuse, overdevelopment, ou encore d'unsustainable use (Custodio, 2000, p. 24). L'ouvrage de Llamas et Custodio (2002) tente d'imposer le terme d'usage intensif (intensive use) comme substitut à tous les autres termes, cette notion faisant apparaître de façon plus explicite le caractère disproportionné des prélèvements réalisés par rapport aux besoins réels des usagers (voir également Llamas, 2002).
} 
« [b]ien des auteurs considèrent que, à strictement parler, la surexploitation survient lorsque les prélèvements sont plus importants ou proches de la recharge moyenne, si des valeurs moyennes de long terme sont prises en compte. Cependant, à la fois les prélèvements et la recharge sont des termes difficiles à définir, particulièrement dans les pays aux climats arides et semi-arides ${ }^{7}$ ». En revanche, ces auteurs préfèrent adopter une définition qui repose sur une évaluation des coûts et des bénéfices engendrés par différents niveaux d'exploitation : «La surexploitation d'un aquifère apparaît lorsque les coûts économiques, sociaux et environnementaux qui dérivent d'un certain niveau de prélèvements sont plus importants que ses bénéfices ${ }^{8}$. » Cette dernière définition pose, elle aussi, des problèmes d'évaluation, mais cette fois d'ordre économique. Comment évaluer les coûts et les bénéfices liés à différents niveaux d'exploitation? Cette évaluation ne risque-t-elle pas de donner plus d'importance à la dimension économique de court terme au détriment de la dimension environnementale qui repose davantage sur le long terme?

L'ensemble de ces questions nous invite à nous tourner vers la définition formulée par l'Association internationale des hydrogéologues (IAH) qui estime qu'un ensemble de critères concourent à une gestion durable des eaux souterraines. Villaroya (1994) avance dans son article les critères suivants :

- la gestion durable des eaux souterraines sur le plan quantitatif : à long terme une nappe ne devrait pas être exploitée au-delà des limites posées par son renouvellement. Des surexploitations de court terme pour les ressources renouvelables peuvent être une méthode économique et acceptable pour l'usage de l'eau dans certains cas spécifiques. L'environnement doit être reconnu comme une demande légitime pour les ressources en eaux souterraines : le maintien des écosystèmes est une nouvelle contrainte de gestion ;

- la gestion durable des eaux souterraines sur le plan qualitatif. Les seules vraies méthodes durables de gestion de la qualité des eaux souterraines sont celles qui sont basées sur le concept de prévention ;

- la gestion doit être intégrée en termes de quantité et de qualité, et prendre en compte le principe d'unicité de la ressource;

\footnotetext{
${ }^{7}$ Hernandez-Mora et al. (2001, p. 111), notre traduction : "Many authors consider that, strictly speaking, over-exploitation occurs when abstraction is greater than or close to average recharge if long-term mean values are taken into account. However, both abstraction and recharge are difficult to define, particularly in arid and semi-arid countries."

8 Ibid., p. 111, notre traduction: "[...] aquifer over-exploitation occurs when the economic, social and environmental costs that derive from a certain level of water abstraction are greater than its benefits."
}

- les usagers de la nappe doivent être informés et éduqués sur l'utilisation de l'eau souterraine et sa protection.

Ces critères nous semblent mieux circonscrire les enjeux de gouvernance des eaux souterraines, car ils mettent en évidence des conditions nécessaires d'ordre plus pragmatique, même si les problèmes d'évaluation des quantités prélevées et rechargées soulignés par Hernandez-Mora et al. (2001) demeurent.

\section{Au-delà de la dichotomie entre marché et État}

D'un point de vue pragmatique, il semble nécessaire d'offrir des solutions à la surexploitation des eaux souterraines qui répondent explicitement à un ensemble de questions : comment assurer, sur le plan quantitatif, la disponibilité des eaux souterraines pour l'ensemble des usages concurrents, sans remettre en cause la pérennité du développement des activités économiques? Comment respecter l'équité entre les usages et assurer le maintien d'un stock de ressources suffisant pour que l'eau souterraine remplisse ses fonctions environnementales? De plus, comment éviter que des conflits d'usage se manifestent et quels mécanismes institutionnels instaurer pour arbitrer entre les usages dans les cas où des conflits seraient avérés? Ces questions rassemblent finalement une conception de l'exploitation des eaux souterraines dans une perspective de développement durable, c'est-à-dire un développement qui satisfait les besoins du présent, sans compromettre la capacité des générations futures à satisfaire leurs propres besoins (CMED, 1989), la dimension intergénérationnelle étant présente dans la notion même de conservation que nous avons mise en avant.

Répondre à l'ensemble de ces questions nécessite de se situer à la fois sur le plan de la gestion de court terme et sur celui de la planification. Par ailleurs, il semble nécessaire de mettre en avant des préoccupations touchant aux critères d'équité et d'efficacité dans l'allocation et la gestion des ressources en eaux souterraines. Face à ce double souci, plusieurs configurations sont envisageables suivant le degré de centralisation ou de décentralisation des choix.

D'une part, certains auteurs ${ }^{9}$ avancent que le marché permettrait d'assurer une allocation efficace des ressources, tout en résolvant les conflits d'usage grâce à la détermination d'un prix de l'eau s'imposant à tous et reflétant la rareté relative des ressources. Rappelons que le terme «marché de l'eau » fait référence majoritairement, sinon exclusivement, à des transferts permanents ou temporaires d'eau entre plusieurs entités géographiques ou

9 Voir notamment Anderson et Snyder (1997). 
administratives (États, régions, bassins et sous-bassins) ou encore sectorielles (transferts de l'agriculture vers l'industrie). Saliba et Bush (1987) caractérisent un marché de droits d'eau de la façon suivante :

- le bien échangé correspond à une quantité d'eau ;

- il existe un acheteur et un vendeur et chacune des deux parties tire un bénéfice de l'échange ;

- le prix de vente du marché de droits d'eau est négocié et non pas fixé de manière exogène. Ceci permet notamment au vendeur de tirer profit de la vente d'eau.

Cette solution nécessite cependant que les droits de propriété privés soient préalablement définis et respectés par l'ensemble des usagers. Or, dans de très nombreux pays, la législation ne permet pas de reconnaître des droits de propriété privés cessibles de manière temporaire ou définitive. Par ailleurs, certaines expériences de marchés de l'eau ne se sont pas révélées aussi efficaces que les prédictions de la théorie économique (fuites dans les canaux d'irrigation, coûts de transaction et d'accès à l'information élevés, dérives monopolistiques... ). Les cas de marchés de l'eau au Chili et aux îles Canaries sont assez exemplaires de ce point de vue, car ils sont souvent présentés par les économistes de la Banque mondiale (Thobani, 1995; Simpson et Ringskog, 1997) comme des réussites et des exemples à suivre alors même que l'efficacité de ces marchés n'a nullement été démontrée ${ }^{10}$.

D'autre part, certains ${ }^{11}$ affirment que l'État doit nécessairement jouer un rôle dans l'allocation, la répartition et la protection des ressources en eau, en vertu de son statut de garant de l'intérêt général. L'allocation et la gestion seraient alors confiées à une agence dont le statut particulier lui permettrait de satisfaire les besoins concurrents, dans le respect de l'équité entre usagers, tout en privilégiant une optique de long terme. Dans les faits, les États, régions ou agences de bassin ont recours à trois types d'instruments pour faire face aux prélèvements excessifs dans les eaux souterraines : instruments que nous pouvons qualifier de " réglementaires », « économiques » et " participatifs ${ }^{12}$. Ces instruments ne peuvent fonctionner de

\footnotetext{
10 Voir à ce sujet les travaux de Bauer $(1997,1998)$ sur les marchés de l'eau au Chili et ceux d'Aguilera-Klink (2002) aux îles Canaries.

11 Voir notamment de Marsily (2002).

12 En référence à Froger et Méral (2002) qui distinguent, dans le cadre des politiques d'environnement, plusieurs formes d'action collective (hiérarchique, incitative et participative). Relevons au sujet de ces trois catégories que les découpages opérés par les économistes pour qualifier les instruments $\mathrm{d}^{\prime}$ " incitatifs/économiques » ou de «réglementaires/hiérarchiques » sont discutables. Certains instruments, comme les quotas, sont parfois classés parmi les instruments réglementaires, parfois parmi les instruments économiques. Nous avons pour notre part classé les quotas parmi les instruments réglementaires, car, dans le domaine de l'irrigation, les quotas sont la plupart du temps appliqués individuellement et ne donnent pas
}

manière efficace qu'à partir du moment où un système de suivi de l'état qualitatif et quantitatif des eaux souterraines est mis en place. Or, force est de constater que la plupart des pays ne sont pas dotés de tels réseaux de mesure, ce qui limite $l^{\prime}$ impact des politiques ${ }^{13}$. Au niveau de l'évolution des instruments, si les moyens réglementaires (permis, quotas, autorisations, interdictions) continuent d'être largement utilisés, le recours aux instruments économiques incitatifs (redevances) et à la participation des usagers encadrée par les pouvoirs publics se développe. Cependant, le niveau des redevances semble encore trop faible pour jouer un rôle incitatif sur les consommations et un certain nombre de politiques publiques combinent des instruments réglementaires, économiques et participatifs ${ }^{14}$.

Le fonctionnement réel des systèmes d'allocation et de gestion des ressources en eaux souterraines est cependant bien différent des deux idéaux types que forment le marché et l'État. Barraqué (2001, pp. 1-2) va dans ce sens lorsqu'il affirme :

«En tout état de cause, le caractère inter-territorial des politiques de l'eau, qui est typique des politiques de l'environnement, et la complexité technique des eaux souterraines (on modélise quelque chose qu'on ne voit même pas, à l'inverse des eaux de surface), rendent particulièrement approprié de choisir des politiques plus négociées et subsidiaires qu'autoritaires; elles seraient conduites à des échelles intermédiaires de gouvernement et en privilégiant la réunion de communautés d'usagers (des usagers différenciés, et non pas des égaux). Autant dire qu'en Europe, l'évolution organisationnelle et institutionnelle de ces dernières décennies ne va dans le sens ni d'une étatisation pure et simple, ni de marchés de droits chers aux économistes de l'environnement. »

Parfois, la propriété publique des eaux souterraines se confond de fait avec une situation de libre accès. Il existe en réalité une très large gamme de formes hybrides combinant à la fois des éléments d'intervention publique et de régulation marchande. En outre, certains systèmes reposent sur des règles de répartition de la ressource

nécessairement lieu à un transfert. Dès lors que les quotas deviennent transférables, ils relèvent davantage des instruments économiques. Tout ceci tend à montrer que l'instrument en lui-même ne relève pas d'une logique mais peut fort bien être adapté à une pluralité de logiques (réglementaire, économique, participative).

13 En France, si de tels réseaux de mesure existent à l'échelle régionale depuis de nombreuses années, la centralisation des données au sein d'un réseau commun de mesure opérant à l'échelon national se met en place progressivement.

14 Notons au sujet des Schémas d'aménagement et de gestion des eaux, qui figurent parmi les instruments participatifs mis en place en France, que la lenteur de la procédure administrative peut conduire, parallèlement, à l'émergence d'initiatives locales de nature plus volontaire (contrats de nappe, initiatives Ferti-Mieux ou Irri-Mieux, par exemple) qui apparaissent moins contraignantes aux acteurs. 
se situant au-delà des formes précédemment citées. Ces systèmes permettent, à l'aide de mécanismes plus ou moins formels d'arrangements entre les usagers, d'allouer les ressources en eau disponibles sans mettre en danger les approvisionnements futurs. Par ailleurs, l'allocation des ressources en eau, dans la très grande majorité des systèmes irrigués de surface, relève d'une logique de l'intérêt public, de l'intérêt privé ou de l'intérêt de l'ensemble de la communauté des usagers. On retrouve cette distinction dans la plupart des articles et ouvrages qui traitent des formes d'allocation de l'eau d'irrigation (Dinar et al., 1997; Ruf et Mathieu, 2001). Notre recherche nous révèle cependant que, comme pour les eaux de surface, la structure des droits de propriété et la configuration particulière de chacune des situations rencontrées dépassent cette distinction. Il existe une multiplicité d'arrangements institutionnels qui combinent plusieurs de ces logiques. Les modes combinant des éléments propres à une allocation communautaire et coexistant avec une régulation publique ou marchande des ressources en eau sont également nombreux. De ce fait, les instruments de marché peuvent fort bien être mobilisés par le régulateur public ou par la communauté des usagers si celle-ci opte pour cette forme d'allocation des ressources en eau ${ }^{15}$.

Cette idée est assez proche du travail précurseur mené sur la gestion patrimoniale des eaux souterraines par Ollagnon (1989), même si celui-ci s'intéresse essentiellement aux problèmes qualitatifs. Il reconnaît notamment le caractère transappropriable des eaux souterraines et insiste sur la nécessité $d$ 'appréhender de manière complémentaire les diverses approches (économique, écologique et technico-administrative). Il insiste notamment, dans un paragraphe portant sur les différents modes de gestion et le problème de la transappropriabilité, sur le fait que « la connaissance du comportement et de la stratégie de "l'acteur-en-relation-avec-son-milieu-naturel-ethumain" requiert une "écologie de l'action" appropriée à la solution des problèmes d'aujourd'hui » (ibid., p. 263). Les concepts doivent être opérationnels et confrontés au réel, dans le but de servir dans le cadre d'une rechercheaction. C'est sans doute sur ce dernier point que notre travail se distingue de celui d'Ollagnon. En effet, si nos résultats sont relativement proches, la démarche d'Ollagnon, qui vise explicitement à formuler des recommandations aux acteurs (par un audit patrimonial) afin qu'ils internalisent la problématique de la qualité comme un «bien commun », va beaucoup plus loin que la nôtre.

Notre démarche, comme nous allons le voir, mobilise le concept de gouvernance pour offrir une vision pragmatique et analytique des situations observables en prenant acte de la nécessité de dépasser la dichotomie entre le

\footnotetext{
15 Voir notamment Strosser (1997) pour le cas des marchés de l'eau au Pakistan.
}

marché et l'État pour l'allocation et la gestion des eaux souterraines.

\section{Un cadre d'analyse pour la gouvernance des eaux souterraines et de l'irrigation}

La gouvernance constitue sans nul doute, à côté du développement durable (Jollivet, 2001), une des expressions les plus utilisées du vocabulaire sociopolitique contemporain. Ceci tient à un flou sémantique fort qui permet d'employer cette expression pour signaler un changement dans la manière de gouverner (dépassement d'une vision purement hiérarchique), mais surtout pour recommander une décentralisation de la gestion des ressources, celle-ci pouvant fort bien s'accommoder d'une régulation marchande ou d'une gestion communautaire des ressources située hors de la sphère des échanges marchands. Afin de prendre en compte cette polysémie et d'offrir une définition qui balise notre champ de réflexion, nous concevons la gouvernance comme relevant d'un compromis entre la coordination des actions individuelles et les formes de l'action collective.

Les notions d'action collective et de coordination des actions individuelles, qui interviennent dans l'étude des processus collectifs, renvoient à la fois à des modes de pensée et à des préconceptions épistémologiques distinctes. Elles postulent en effet un rapport de l'individuel à la collectivité qui transparaît dans la définition qu'offre Weinstein (1997, p. 390) de la coordination :

«Par mode de coordination, il faut entendre les différentes procédures qui concourent à la compatibilité des comportements d'agents en interaction et permettent d'atteindre un certain degré d'efficience [...] dans l'obtention de résultats dépendants des actions de ces agents. La coordination se présente comme une succession d'actions individuelles en interaction ; toute procédure de coordination ayant pour effet d'orienter et de contraindre les actions, donc de limiter le champ des actions possibles.»

Cette vision de la coordination qui donne le primat à l'individu sur le social permet de montrer la filiation existant entre cette notion et l'individualisme méthodologique.

À l'opposé, l'action collective trouve ses racines dans une conception holiste des rapports entre l'individu et la société. Les développements récents de l'analyse économique ont montré, selon nous, que deux courants principaux offrent un point de vue convergent sur 1'action collective, qui permet de dépasser la dichotomie habituellement opérée entre le marché et l'État.

Le premier de ces courants dispose de fondements théoriques et épistémologiques assez anciens et s'est développé dans le sillage des travaux des institutionnalistes américains du début du siècle dernier (Veblen, Commons 
et Mitchell). Les institutionnalistes qui prennent l'environnement pour champ d'investigation ${ }^{16}$ empruntent à leurs aînés une perspective holiste, systémique et évolutionniste qui leur permet de souligner la nécessité d'une coévolution durable des sphères économique, sociale et naturelle. Ces auteurs appellent à une action collective orientée vers un compromis entre l'intervention publique et le marché, reposant sur la participation et la démocratie. En s'appuyant sur la philosophie pragmatique américaine, les institutionnalistes développent une approche méthodologique qui modifie le rapport établi entre les chercheurs et les objets réels qu'ils étudient (principe d'abduction ou théorie de l'enquête de Dewey). Cette approche pragmatique s'adapte assez bien au souci de réalisme que nous mettons en avant et mérite donc toute notre attention ${ }^{17}$.

Le second courant, qui forme selon nous un prolongement du premier, s'intéresse aux modalités d'allocation et de gestion des ressources communes. À partir d'un certain nombre d'études de cas de ressources communes soigneusement sélectionnées, comme les bassins aquifères, les pêcheries, les forêts ou les systèmes irrigués, les auteurs du courant de la propriété commune rejettent l'interprétation pessimiste défendue par Hardin et soulignent, au contraire, la capacité des individus à restructurer leur propre situation interdépendante (Ostrom, 1990 ; Bromley, 1992 ; Burger et al., 2001). Un des axes majeurs d'analyse repose sur l'idée qu'il existe une grande variété de situations et de problèmes d'action collective, mais qu'il existe également une large gamme de solutions. Le message important est que chaque groupe peut s'organiser et s'engager dans un travail de restructuration des institutions pour répondre à chaque problème spécifique. L'analyse de l'action collective balance donc d'un pan théorique à un terrain plus pragmatique d'expérimentation. Cette approche, qui s'attache par exemple à l'étude du fonctionnement traditionnel de l'allocation des ressources d'un point de vue communautaire, peut être riche d'enseignements pour comprendre les modes de gouvernance reposant sur la participation et la concertation qui sont en plein essor actuellement, mais dont les racines peuvent parfois être très anciennes ${ }^{18}$.

Ces deux courants permettent d'offrir, de manière complémentaire, un éclairage sur les solutions collectives

\footnotetext{
16 Voir Swaney (1987) ou, pour une synthèse plus récente, Petit (2002a).

17 Notons au passage que l'approche développée par Ollagnon (1989) est parfaitement conciliable avec cette démarche méthodologique qui peut conduire le chercheur à une posture de participant-observateur.

${ }^{18}$ Le lien entre les communautés d'usagers d'eau souterraine existant actuellement en Espagne et les huertas traditionnelles du sud-est espagnol, opérant sur les eaux de surface, est un bon exemple de rapprochement (voir Petit, 2002b).
}

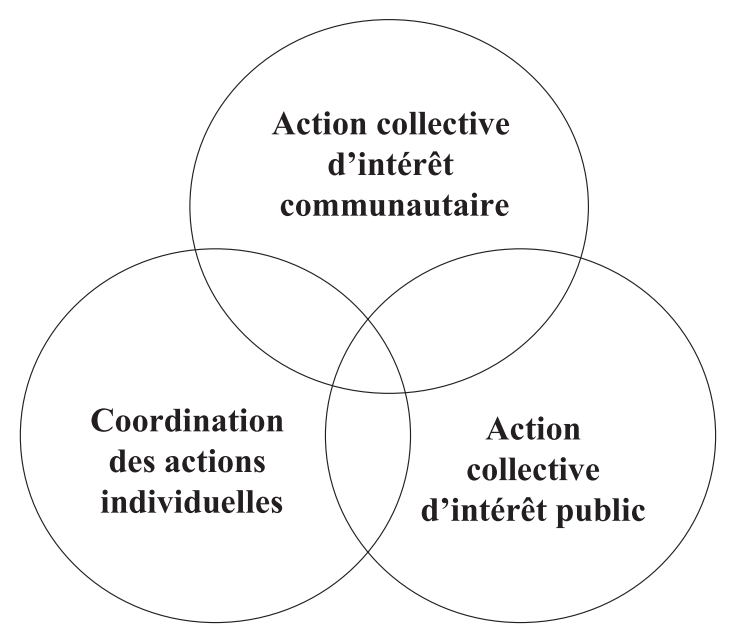

Fig. 1. Le schéma de la gouvernance.

et coopératives adoptées par les acteurs se partageant une ressource commune.

Afin d'approfondir notre distinction entre coordination des actions individuelles et formes de l'action collective, soulignons que, dans l'optique de notre définition de la gouvernance, la coordination des actions individuelles désigne le mode d'organisation des relations qui s'établissent entre les acteurs/agents économiques, dans le cadre d'un marché. En contraste, nous pouvons définir l'action collective comme la recherche par un groupe déterminé d'acteurs/agents d'une procédure formelle ou informelle de résolution d'un problème commun.

Suivant les acteurs/agents concernés et le degré formalisé ou non des procédures, l'action collective pourra recouvrir deux formes distinctes que nous dénommons « action collective d'intérêt public » et «action collective d'intérêt communautaire » (Fig. 1).

L'action collective d'intérêt public est formée de l'ensemble des procédures mises en place par la puissance publique ou l'État. Cette forme d'action collective mobilise des instruments réglementaires, économiques et négociés qui renvoient aux formes hiérarchiques, incitatives et participatives recensées par Froger et Méral (2002). L'action collective d'intérêt communautaire regroupe quant à elle l'ensemble des procédures collectives mises en place par les acteurs/agents, en lien ou indépendamment des procédures de régulation publique ou marchande. Cette forme d'action collective se caractérise par la tentative de conciliation d'intérêts parfois contradictoires, dans une démarche concertée, dépassant les clivages traditionnels entre les acteurs et trouvant sa raison d'être dans l'accomplissement d'un objectif servant les intérêts de la communauté des usagers.

La coordination des actions individuelles et les deux formes d'action collective que nous venons de définir interviennent sur l'allocation et la gestion des ressources en eau. 
Encadré 2. La nappe de Beauce : un enjeu de gouvernance

La nappe de Beauce, située à l'ouest de Paris, s'étend sur un périmètre de près de $9000 \mathrm{~km}^{2}$. Elle concerne six départements (Eure-et-Loir, Loir-et-Cher, Loiret, Seine-et-Marne, Yvelines, Essonne), deux régions (Centre et Île-de-France) et chevauche deux grands bassins (Loire-Bretagne et Seine-Normandie). Les sécheresses successives survenues à la fin des années 1980 et au début des années 1990, couplées à un accroissement des quantités d'eau prélevées pour l'irrigation, ont contribué à une forte baisse du niveau piézométrique moyen. En effet, de 1986 à 1994, le niveau de la nappe de Beauce est passé de son niveau maximum enregistré à son plus bas niveau historique. Cette situation a provoqué des tensions entre les différentes catégories d'usagers. Pour résoudre cette situation, les prélèvements dans la nappe de Beauce ont fait l'objet, au milieu des années 1990, de mesures préfectorales spécifiques concernant son usage agricole notamment (interdictions d'irriguer certains jours). Parallèlement, suite à des discussions avec la profession agricole et à la signature d'une charte en 1995, des seuils d'alerte concernant le niveau moyen de la nappe ont été mis en place et une gestion par les quotas a été installée par les préfectures. En outre, une procédure de Schéma d'aménagement et de gestion des eaux (SAGE) est en cours depuis 1999, en même temps qu'un certain nombre d'initiatives plus localisées (deux procédures Irri-Mieux lancées à la fin des années 1990 et récemment interrompues) ou touchant des publics ciblés se développent depuis quelques années.

Prenons pour illustration l'évolution de la gouvernance de la nappe de Beauce (Encadré 2) depuis la situation de crise quantitative du début des années 1990. Cette évolution montre une hybridation des deux formes d'action collective ici recensées.

À partir de l'étude des procédures de gestion et des mécanismes de concertation dont la nappe de Beauce a fait l'objet depuis le début des années 1990 (Petit et Corcos, 2001; Petit, 2002b), il apparaît que le processus suit un double mouvement :

- un affermissement du rôle de l'État dans le suivi qualitatif et quantitatif de la nappe de Beauce et dans la mobilisation des instruments réglementaires (systèmes d'autorisation, zones de répartition des eaux, mise en place de quotas), économiques (application des redevances sur les prélèvements et les rejets) et participatifs (SAGE, procédures Irri-Mieux, Ferti-Mieux, etc.);

- une affirmation du rôle des communautés d'usagers au sein des structures de concertation, les instruments participatifs se muant progressivement en des systèmes de décision polycéphale où l'administration et les acteurs publics délèguent une partie de leurs pouvoirs au profit des autres usagers. Cette nouvelle configuration, déjà observable au niveau des grands bassins hydrographiques et même au niveau national, nous invite à faire entrer ces instruments au carrefour de l'action collective d'intérêt public et d'intérêt communautaire.

Ce mouvement ne laisse pour le moment aucune place à la coordination des actions individuelles, même si plusieurs auteurs estiment que le système des quotas mis en place pourrait se transformer en un marché de l'eau dont la Commission locale de l'eau (CLE), noyau opérationnel du SAGE, constituerait la structure porteuse (Kosciusko-Morizet et al., 1999; Strosser et Montginoul, 2001).

Il semble finalement que les eaux souterraines, par leur caractère invisible, leurs droits de propriété toujours ambigus, l'affranchissement des frontières administratives qui les caractérisent souvent, se prêtent mieux que bien d'autres ressources à des formes hybrides de gouvernance. Le caractère transappropriable des eaux souterraines relevé par Ollagnon (op.cit.) peut être interprété dans ce contexte comme une superposition de droits d'usage, mais aussi comme une manière pour chacun des groupes d'acteurs de s'approprier les instruments de l'action.

\section{Conclusion}

La question de la surexploitation des eaux souterraines montre que les schémas habituels de pensée qui reposent sur une stricte dichotomie entre le marché et l'État méritent d'être reconsidérés. C'est souvent à partir de situations de pénurie que les acteurs locaux ressentent le caractère commun des eaux souterraines et tentent de trouver les solutions les plus adaptées à la configuration juridique, économique et politique qui définit leur rapport à la ressource. Or, les perspectives de croissance démographique à l'horizon $2050^{19}$ font craindre une intensification des prélèvements dans les eaux de surface comme dans les eaux souterraines. Même les pays en développement les mieux dotés en eau, comme l'Inde, connaissent déjà des situations locales de surexploitation de leurs aquifères. Face à l'aggravation de cette crise de l'eau à l'échelle mondiale, les dispositifs réglementaires internationaux demeurent insuffisants. Il n'existe pour le moment aucune convention d'environnement sur les ressources en eau, même si les conventions sur la diversité biologique, les changements climatiques et la désertification intègrent déjà des volets touchant à cette question.

\footnotetext{
19 Un récent rapport des Nations Unies (http://un.org/esa/ population/pop.htm) estime que la population mondiale devrait atteindre 8,9 milliards d'habitants en 2050, les deux pays les plus peuplés demeurant la Chine (avec 1,395 milliard) et l'Inde (avec 1,531 milliard).
} 
Pourtant, au regard du nombre de conférences internationales sur ce sujet (de Mar del Plata en 1977 à Kyoto en 2003), il semble que la communauté internationale ait pris la mesure du danger qui la guette.

Notre étude de la gouvernance des eaux souterraines relève une transition qui s'est opérée entre une gestion publique ou marchande et une gestion concertée de ces ressources. Un tel schéma s'avère lui aussi nécessaire pour que la coopération internationale puisse garantir une répartition équitable de ces ressources, disponibles en quantité finie.

\section{Remerciements}

Je souhaite remercier les relecteurs de la revue Natures Sciences Sociétés, et en particulier Franck-Dominique Vivien et Jean Margat pour leurs commentaires sur une version antérieure de cet article. Je reste bien évidemment seul responsable des erreurs subsistantes.

\section{Références}

Adour, Garonne, 2002. Hors série, Spécial Eaux souterraines, Adour Garonne, revue de l'Agence de l'eau, hiver.

Aguilera-Klink, F., 1994. Some Notes on the Misuse of Classic Writings in Economics on the Subject of Common Property, Ecological Economic, 9, 3, April, 221-228.

Aguilera-Klink, F., 2002. Los mercados de agua en Tenerife, Bilbao, Bakeaz.

Anderson, T.L., Snyder, P.S., 1997. Water Markets. Priming the Invisible Pump, Washington DC, CATO Institute.

Barraqué, B., 2001. Nouvelles politiques de gestion des eaux souterraines en Europe, notamment concernant la pollution diffuse, in Commissariat général du plan (Ed.), La Politique de protection des eaux destinées à l'alimentation humaine, Paris, La Documentation française.

Bauer, C.J., 1997. Bringing Water Markets down to Earth: The Political Economy of Water Rights in Chile, 1976-95, World Development, 25, 5, 639-656.

Bauer, C.J., 1998. Slippery Property Rights: Multiple Water Uses and the Neoliberal Model in Chile, 1981-1995, Natural Resources Journal, 38, 109-155.

Bromley, D.W. (Ed.), 1992. Making the Commons Work: Theory, Practice and Policy, San Francisco, ICS Press.

Burger, J., Ostrom, E., Norgaard, R.B., et al., 2001. Protecting the Commons. A framework for Resource Management in the Americas, Washington DC, Island Press.

Ciriacy-Wantrup, S.V., Bishop, R.C., 1975. «Common Property » as a Concept in Natural Resources Policy, Natural Resources Journal, 15, October, 713-727.

CMED, 1989. Notre avenir à tous, Montréal, Éditions du Fleuve.

Custodio, E., 2000. The complex concept of overexploited aquifer, in Llamas, R.M. (Ed.), Papeles del proyecto aguas subterráneas, Madrid, Fundacion Marcelino Botin.

Dinar, A., Rosegrant, M.W., Meinzen-Dick, R.S., 1997. Water Allocation Mechanisms: Principles and Examples, Washington DC, World Bank.
European Environment Agency, 1999a. Groundwater Quality and Quantity in Europe, Luxembourg, Office for Official Publications of the European Communities.

European Environment Agency, 1999b. Sustainable Water Use in Europe. Part 1: Sectoral Use of Water, Luxembourg, Office for Official Publications of the European Communities.

Foster, S., 2000. Sustainable Groundwater Exploitation for Agriculture. Current Issues and Recent Initiatives in the Developing World, in Llamas, R.M. (Ed.), Uso Intensivo de Las Aguas Subterraneas, Aspectos éticos, technologicos y economicos, Madrid, Fundacion Marcelino Botin.

Froger, G., Méral, P., 2002. Des mécanismes de l'action collective aux perspectives pour les politiques d'environnement, in Froger, G., Méral, P. (Eds.), Gouvernance 2. Action collective et politique d'environnement, Bâle, Genève, Helbing \& Lichtenhahn, 9-24.

Grellet, G., 2001. Systèmes d'irrigation et droits de propriété, Revue Tiers monde, 42, 166, 317-331.

Hardin, G., 1968. The Tragedy of the Commons, Science, 162, 1243-1248.

Hernandez-Mora, N., Llamas, R.M., Martinez Cortina, L., 2001. Misconceptions in Aquifer Over-Exploitation: Implications for Water Policy in Southern Europe, in Dosi C. (Ed.), Agricultural Use of Groundwater. Towards Integration Between Agricultural Policy and Water Resources Management, Dordrecht, Kluwer and Fondazione Eni Enrico Mattei, 107-126.

Jollivet, M., 2001. Introduction, in Jollivet M. (Ed.), Le Développement durable, de l'utopie au concept. De nouveaux chantiers pour la recherche, Paris, Elsevier, 9-18.

Kosciusko-Morizet, N., Richard,V., Lamotte, H., 1999. Que peuton attendre de la mise en place de quotas individuels échangeables de prélèvements sur la ressource en eau en France? L'exemple de l'agriculture irriguée, in Falque, M., Massenet, M. (Eds.), Droits de propriété, économie et environnement. Les ressources en eau, Paris, Dalloz, 175-198.

Llamas, R.M., 2002. Intensive use of groundwater, Third World Water Pre-forum, Toyama.

Llamas, R.M., Custodio, E. (Eds.), 2002. Intensive Use of Groundwater: Challenges and Opportunities, The Netherlands, Swets and Zeitlinger.

Marsily, G. (de), 2002. Vers des parcs naturels hydrologiques, Manière de voir, Le Monde diplomatique, 65, 88-91.

Ollagnon, H., 1989. Une approche patrimoniale de la qualité du milieu naturel, in Mathieu, N., Jollivet, M. (Eds.), Du rural à l'environnement. La question de la nature aujourd'hui, Paris, ARF/L'Harmattan, 258-268.

Ostrom, E., 1990. Governing the Commons. The Evolution of Institutions for Collective Action, Cambridge, Cambridge University Press.

Petit, O., 2002a. Action collective et politiques d'environnement orientées vers une coévolution durable : le regard des institutionnalistes, in Froger, G., Méral, P. (Eds.) Gouvernance 2. Action collective et politique d'environnement, Bâle, Genève. Helbing \& Lichtenhahn, 25-45.

Petit, O., 2002b. De la coordination des actions individuelles aux formes de l'action collective : une exploration des modes de gouvernance des eaux souterraines. Thèse de doctorat en Sciences économiques, Université de Versailles-Saint-Quentin-enYvelines, décembre.

Petit, O., Corcos, B., 2001. Appropriation sociale du problème de gestion durable de la nappe de Beauce, Rapport pour le Programme inter-institutionnel de recherches et d'études 
en économie de l'environnement (PIREE), sous la direction scientifique de M. O'Connor, C3ED, Ministère de l'Aménagement du territoire et de l'Environnement, août.

Ruf, T., Mathieu, P., 2001. Introduction. Water Rights and the Institutional Dynamics of Irrigated Systems: between State, market and community action, International Journal of Water, 1, 3-4.

Saleth, R.M., 1996. Water Institutions in India, New Delhi, Comonwelth Publishers.

Saliba, B.G., Bush, D.B., 1987. Water marketing in the Southwest - Can market prices be used to evaluate supply augmentation projects?, Technical Bulletin, Washington DC, US Foreign Services.

Shiklomanov, I., 1991. The World's Water Resources, International Symposium to Commemorate the 25 Years of IHD/IHP, Paris, Unesco.

Shiklomanov, I., 1993. World Freshwater Resources, in Gleick, P. (Ed.), Water in Crisis, Oxford, Oxford University Press, $1-24$.

Simpson, L., Ringskog, K., 1997. Water Markets in the Americas, Report $\mathrm{n}^{\circ} 17282$, Directions in Development Series, Washington DC, World Bank.
Strosser, P., 1997. Analysing Alternative Policy Instruments for the Irrigation Sector. An assessment of the potential for water market development in the Chishtian Sub-division, Wageningen Agricultural University, Ph.D. Thesis, Wageningen.

Strosser, P., Montginoul, M., 2001. Vers des marchés de l'eau en France? Quelques éléments de réflexion, Annales des Mines, Responsabilité et Environnement, juillet, 13-31.

Swaney, J.A., 1987. Elements of a Neoinstitutional Environmental Economics, Journal of Economic Issues, 21, 4, 1739-1779.

Thobani, M., 1995. Tradable Property Rights to Water. How to improve water use and resolve water conflicts, Public Policy for the Private Sector, February.

Villarroya, F., 1994. Regulatory issues mainly about aquifer overexploitation within the scope of sustainable development, Future Groundwater Resources at Risk, Proceedings of the Helsinki Conference, 222, 389-401.

Weber, J., Réveret, J.-P., 1994. Biens communs : les leurres de la privatisation, in Une terre en renaissance, Le Monde diplomatique, collection Savoirs, 2, 71-73.

Weinstein, O., 1997. Modèles d'innovation et modes de coordination inter et intra-entreprises, in Palloix C., Rizopoulos Y. (Eds.), Firmes et économie industrielle, Paris, L'Harmattan, 389-413.

Reçu le 12 juin 2003. Accepté le 6 novembre 2003.

To access this journal online: www.edpsciences.org 